\title{
Direct Evidence of Acetaminophen Interference with Subcutaneous Glucose Sensing in Humans: A Pilot Study
}

\author{
Ananda Basu, MD, Sona Veettil, MD, Roy Dyer, $\mathrm{PhD}^{2}$ Thomas Peyser, $\mathrm{PhD}^{3}$ \\ and Rita Basu, MD'
}

\begin{abstract}
Background: Recent advances in accuracy and reliability of continuous glucose monitoring (CGM) devices have focused renewed interest on the use of such technology for therapeutic dosing of insulin without the need for independent confirmatory blood glucose meter measurements. An important issue that remains is the susceptibility of CGM devices to erroneous readings in the presence of common pharmacologic interferences. We report on a new method of assessing CGM sensor error to pharmacologic interferences using the example of oral administration of acetaminophen.

Materials and Methods: We examined the responses of several different Food and Drug Administration-approved and commercially available CGM systems (Dexcom [San Diego, CA] Seven ${ }^{\circledR}$ Plus ${ }^{\mathrm{TM}}$, Medtronic Diabetes [Northridge, CA] Guardian ${ }^{\circledR}$, and Dexcom G $4{ }^{\circledR}$ Platinum) to oral acetaminophen in 10 healthy volunteers without diabetes. Microdialysis catheters were placed in the abdominal subcutaneous tissue. Blood and microdialysate samples were collected periodically and analyzed for glucose and acetaminophen concentrations before and after oral ingestion of $1 \mathrm{~g}$ of acetaminophen. We compared the response of CGM sensors with the measured acetaminophen concentrations in the blood and interstitial fluid.

Results: Although plasma glucose concentrations remained constant at approximately $90 \mathrm{mg} / \mathrm{dL}$ (approximately $5 \mathrm{mM}$ ) throughout the study, CGM glucose measurements varied between approximately 85 to $400 \mathrm{mg} / \mathrm{dL}$ (from approximately 5 to $22 \mathrm{mM}$ ) due to interference from the acetaminophen. The temporal profile of CGM interference followed acetaminophen concentrations measured in interstitial fluid (ISF).

Conclusions: This is the first direct measurement of ISF concentrations of putative CGM interferences with simultaneous measurements of CGM performance in the presence of the interferences. The observed interference with glucose measurements in the tested CGM devices coincided temporally with appearance of acetaminophen in the ISF. The method applied here can be used to determine the susceptibility of current and future CGM systems to interference from acetaminophen or other exogenous pharmacologic agents.
\end{abstract}

\section{Introduction}

A FUNDAMENTAL REQUIREMENT for a safe and effective artificial pancreas system is an accurate and precise continuous glucose monitoring (CGM) system to measure interstitial fluid (ISF) glucose concentrations and provide input to a control algorithm, which in turn determines insulin dosing through an insulin delivery device (i.e., insulin pump). Recent improvements in the accuracy and reliability of
Dexcom (San Diego, CA) G4 ${ }^{\circledR}$ Platinum CGM systems have focused renewed attention on the feasibility of commercializing artificial pancreas devices. ${ }^{1}$ The two leading manufacturers of CGM devices, Medtronic Diabetes (Northridge, CA) and Dexcom, have recently released new generations of CGM devices with markedly improved accuracy compared with earlier generation devices., ${ }^{2,3}$

In addition to their use as a critical component in research studies of future artificial pancreas devices, CGM devices are

\footnotetext{
${ }^{1}$ Endocrine Research Unit, Saint Mary's Hospital, Mayo Clinic, Rochester, Minnesota.

${ }^{2}$ Immunochemical Core Laboratory, Mayo Clinic, Rochester, Minnesota.

${ }^{3}$ Automated Glucose Control LLC, Palo Alto, California.
} 
also now in increasing use by patients with diabetes. A survey of participants in the T1D Exchange clinic registry in 2014 found that almost $10 \%$ of patients used a CGM device during a 30-day period. ${ }^{4}$ A recent in silico study by Kovatchev et al. ${ }^{5}$ found that CGM systems with a mean absolute relative difference below $10 \%$ were suitable for use in open loop without the need for independent confirmatory blood glucose meter measurements.

Although the new CGM systems have achieved much higher performance standards than previous generations of such devices, the recent improvements in accuracy and reliability of such systems may still be compromised by exogenous pharmacologic interferences. In vitro studies have suggested potential interferences of commonly used overthe-counter medications (acetaminophen, aspirin, and vita$\min C$ ) with glucose sensors that use glucose oxidase- and glucose dehydrogenase-based methods to measure glucose concentrations. $^{6-9}$

Despite the potential serious clinical consequences associated with falsely high or low CGM measurements due to pharmacologic interferences, there have been few investigations of this issue by independent research groups. Recently, Maahs et al. ${ }^{10}$ reported on erroneous high readings in the Dexcom G4 Platinum in subjects with diabetes after administration of acetaminophen. Despite anecdotal reports and well-established theoretical reasons to suspect the possibility of interference from acetaminophen and other substances, there is no generally accepted method for evaluating the impact of pharmacologic interferences on sensor performance.

As CGM systems begin to play a larger role in routine clinical practice and future artificial pancreas systems, there is a critical need for an established methodology with which to evaluate sensors for common drug interferences. In this article we present our findings of the acetaminophen interference on two older-generation CGM systems and one currently extant CGM system, but also a methodology that can be used for future quantitative characterization of sensor performance in the presence of any potential pharmacologic interference.

We have been using the microdialysis technique to measure ISF glucose kinetics as part of other research projects aimed at improving sensor technology. ${ }^{11-13}$ The microdialysis technique allows extraction of metabolites and hormones (e.g., glucose, insulin) from ISF for quantitative measurements. Because CGM measures ISF glucose levels, a reliable methodology to study interference would be to measure pharmaceutical concentrations directly in the microdialysate and establish interference patterns with CGM in humans. To do so, we have applied the microdialysis technique, in combination with CGM devices and ISF drug measurements, to develop an experimental model in humans to demonstrate the direct effects, if any, of pharmaceutical agents on CGM glucose measurements. To our knowledge there are no systematic evaluations being currently conducted to evaluate glucose sensors in this manner in vivo in humans.

This research is a first attempt to systematically and prospectively identify potentially interfering substances (in this case, acetaminophen), to quantify concentrations in plasma and ISF, and to demonstrate interference with ISF glucose sensing. The data thus generated can be directly applied toward improvement of sensor technology. Not only will this enhance the quality and reliability of the sensors, but also will provide valuable guidelines to doctors and patients on effective management of their condition. These results may also be of interest to regulatory bodies assessing the readiness of CGM systems to be used nonadjunctively or as a critical component of artificial pancreas devices.

\section{Materials and Methods}

After approval from Mayo Institutional Review Board and informed consent, 10 healthy nonsmoking subjects without diabetes were enrolled in the study. This study was deliberately done in subjects without diabetes to provide a stable glucose value against which the effect of pharmacologic interferences could be clearly demonstrated. During the screening visit a routine physical examination and appropriate laboratory tests were performed to ensure that subjects were healthy, on no medications, and with normal cardiac, hepatic, and renal functions and had normal fasting blood glucose concentrations. A pregnancy test was performed where appropriate to exclude pregnancy. Enrolled subjects returned to the Mayo Clinical Research Trials Unit (CRTU) within 2 weeks of the screen visit and 2 days prior to the study date for placement of CGM devices. The CGM systems used in the present study were the Guardian ${ }^{\circledR}$ REAL-Time CGM system (Medtronic Diabetes) and the Dexcom Seven ${ }^{\circledR}$ Plus $^{\mathrm{TM}}$ and G4 Platinum CGM systems. At the time of the study, these were the only three Food and Drug Administration-approved and commercially available stand-alone CGM systems.

On the night before the study, subjects reported to the CRTU for admittance, sensors were calibrated as per manufacturers' guidelines, and participants were fed a standard supper at approximately 5 PM. Thereafter, subjects remained fasting other than sips of water until study completion the following day. In the following morning at approximately 7 AM, an intravenous cannula was inserted in a retrograde fashion into a dorsal hand vein. The hand was then placed in a heated plexiglass box (approximately $55 \mathrm{C}^{\circ}$ ) to obtain arterialized venous blood at periodic intervals until 4 PM. At approximately $6 \mathrm{AM}$ four microdialysis catheters were inserted, under local anesthesia and with aseptic precautions into the subcutaneous abdominal fatty tissue. The catheters were flushed to ensure patency. The microdialysis catheters were inserted adjacent to the two CGM devices. Thereafter, a constant infusion of microdialysis perfusate was started through the microdialysis catheters and continued until study end. After an adequate equilibration period, baseline plasma and ISF samples were collected. Thereafter, at $8 \mathrm{AM}$ a dose $(1 \mathrm{~g})$ of acetaminophen (Tylenol ${ }^{\circledR}$; Johnson and Johnson, New Brunswick, NJ) was administered by mouth. At periodic intervals throughout the study, microdialysate and blood samples were collected for glucose and acetaminophen measurements. Following study completion, all cannulae and devices were removed, and the participants were provided a meal and dismissed from the CRTU.

Plasma glucose concentrations were measured with the glucose oxidase method using the YSI analyzer (Yellow Springs Instruments, Yellow Spring, OH). Plasma acetaminophen was measured with a commercial automated assay (ACETA) on the COBAS c311 (Roche Diagnostics, Indianapolis, Indiana). In brief, acetaminophen from a $2.5-\mathrm{mL}$ plasma sample volume was hydrolyzed by an arylacylamidase to yield $p$-aminophenol and acetate. Subsequently the $p$-aminophenol was converted to an 
Table 1. Subject Characteristics

\begin{tabular}{lc}
\hline Characteristic & Value \\
\hline Age (years) & $36 \pm 18$ \\
Sex (male:female) & $2: 8$ \\
Weight (kg) & $74.7 \pm 12.7$ \\
BMI (kg/m ${ }^{2}$ ) & $26.9 \pm 3.3$ \\
Waist-to-hip ratio & $0.87 \pm 0.09$ \\
Fasting plasma glucose (mg/dL) & $84.9 \pm 10.7$ \\
Total body fat (\%) & $35.7 \pm 5.9$ \\
Fat-free mass $(\mathrm{kg})$ & $46.2 \pm 10.3$
\end{tabular}

BMI, body mass index.

indophenol in the presence of $o$-cresol and a periodate catalyst. The production of indophenol was followed colorimetrically $(600 \mathrm{~nm})$. The change in absorbance is directly proportional to the quantitative drug concentration in the sample. The measuring range is $1.2-500 \mu \mathrm{g} / \mathrm{mL}$. The limit of quantitation in plasma is $1.2 \mu \mathrm{g} / \mathrm{mL}(7.94 \mu M)$, defined as the lowest measurable analyte level that can be distinguished from zero. It is calculated as the value lying 2 SDs above that of the $0 \mu \mathrm{g} / \mathrm{mL}$ calibrator (standard $1+2 \mathrm{SD}$, within-run precision, $n=21)$. Intra- and interassay precision at $5.9 \mu \mathrm{g} / \mathrm{mL}(39.1 \mu M)$ is $4.5 \%$ and $6.9 \%$ coefficient of variation, respectively. ISF acetaminophen was also analyzed on the COBAS c311. Because of the small sample volume, approximately $20-40 \mu \mathrm{L}$ of microdialysate was diluted in phosphate-buffered saline to a final volume of $50 \mu \mathrm{L}$ for analysis. The limit of quantitation for microdialysate samples was found to be similar as in plasma samples; intra-assay precision ranged from $0.53 \%$ to $7.7 \%$. Interassay precision was not assessed because all samples were run on a single day.

\section{Results}

\section{Subject characteristics}

The subject characteristics are provided in Table 1. In brief, 10 subjects (two men and eight women; age, $36 \pm 18$ years; weight, $74.7 \pm 12.7 \mathrm{~kg}$; body mass index, $26.9 \pm 3.3 \mathrm{~kg} / \mathrm{m}^{2}$ ) with a normal fasting glucose level of $84.9 \pm 10.7 \mathrm{mg} / \mathrm{dL}$ were enrolled.

\section{Plasma and ISF acetaminophen concentrations (Fig. 1)}

Figure 1 shows the temporal profile of plasma and ISF acetaminophen concentrations following ingestion of $1 \mathrm{~g}$ of acetaminophen at time 0. Acetaminophen concentrations reached a peak within 60-90 $\mathrm{min}$ in the plasma and the ISF and thereafter steadily declined and reached the lower limits of detection within the last hour of study.

\section{CGM and plasma glucose concentrations (Fig. 2)}

Figure 2 shows the temporal profiles of plasma glucose based on CGM glucose readings in all three CGM devices studied following ingestion of $1 \mathrm{~g}$ of acetaminophen at time 0 . As shown, CGM readings from all three devices tested began to rise within $30 \mathrm{~min}$ of ingestion of acetaminophen, rising to a peak concentration before gradually declining toward the end of the study (approximately $7 \mathrm{~h}$ later). The change in CGM readings appeared to coincide with acetaminophen appearing in the ISF, which in turn also coincided with pattern of plasma acetaminophen concentrations. It is noteworthy that plasma glucose concentrations did not change and remained at approximately $90 \mathrm{mg} / \mathrm{dL}$ throughout the study duration in all subjects. The degree of interference with

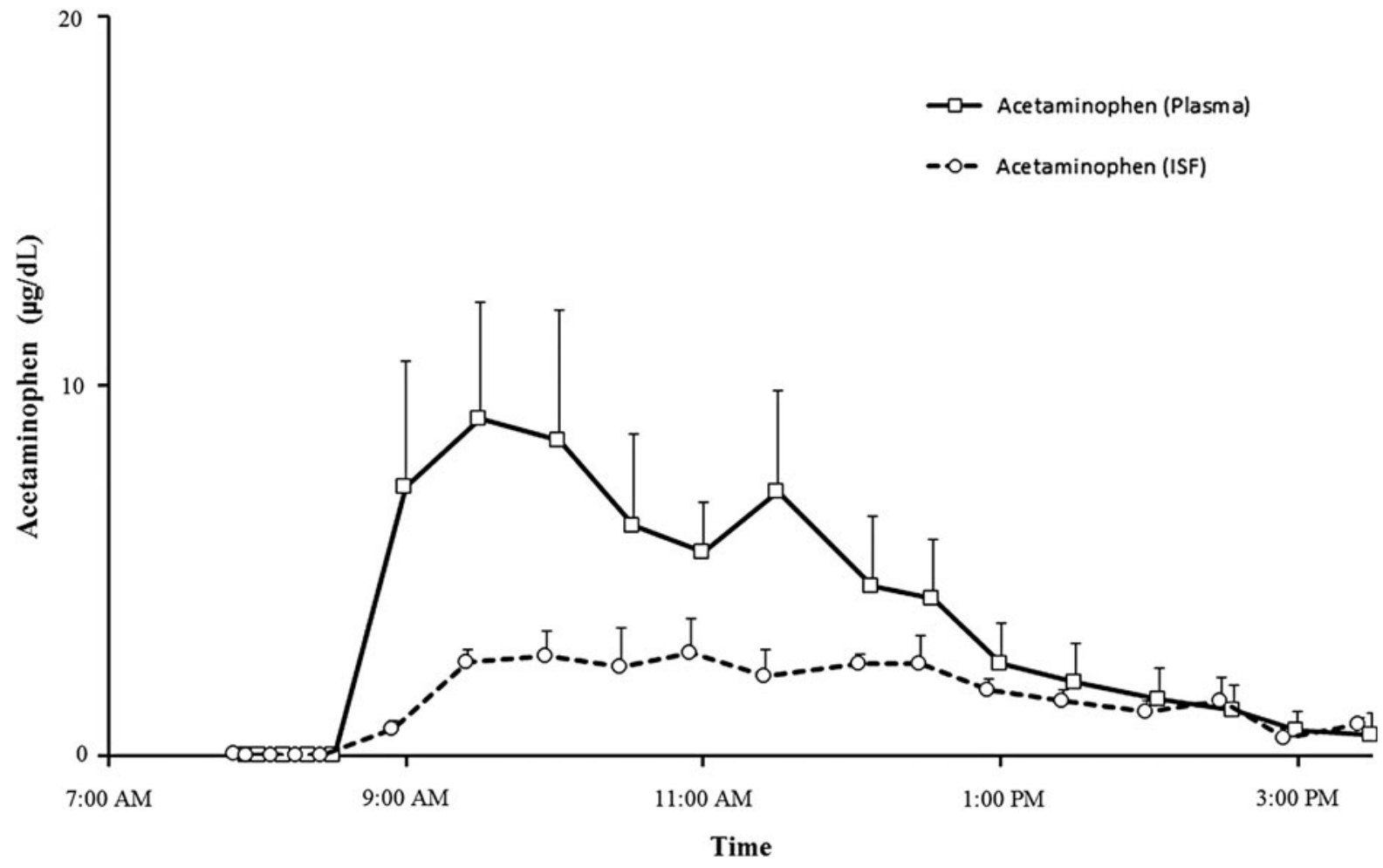

FIG. 1. The temporal profile of plasma and interstitial fluid (ISF) acetaminophen concentrations following ingestion of $1 \mathrm{~g}$ of acetaminophen at time 0 . 


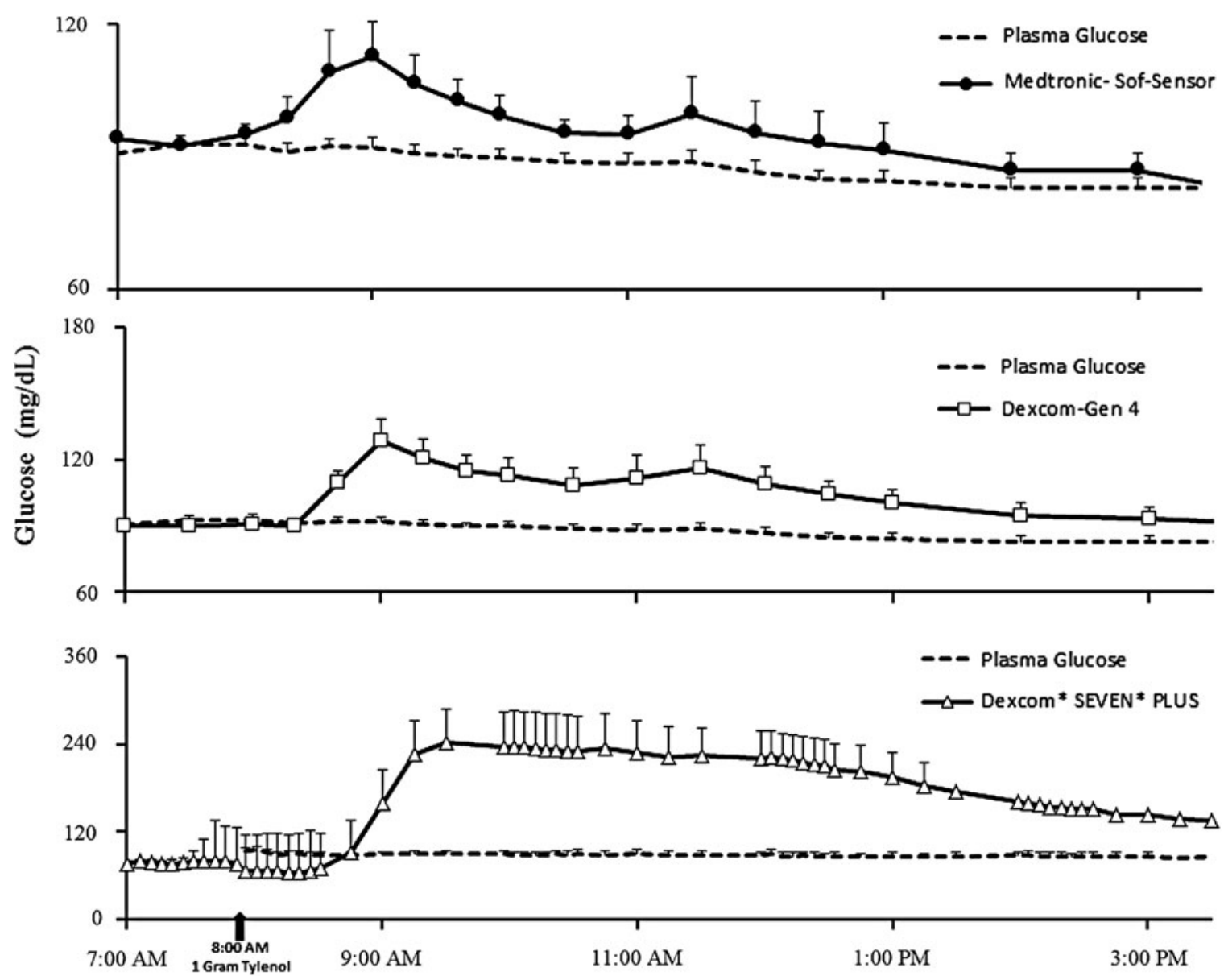

FIG. 2. The temporal profiles of continuous glucose monitoring plasma glucose concentrations for all three devices: (upper panel) Medtronic REAL-Time Guardian, (middle panel) Dexcom G4 Platinum, and (lower panel) Dexcom Seven Plus studied following ingestion of $1 \mathrm{~g}$ of acetaminophen at time 0 .

acetaminophen appeared to be the worst with the older device (Dexcom Seven Plus) than with the G4 Platinum CGM system or the Medtronic REAL-time Guardian.

\section{Discussion}

We have demonstrated direct evidence that ingestion of $1 \mathrm{~g}$ of acetaminophen interferes with CGM glucose readings to different degrees. The greatest magnitude of error was observed with the Dexcom Seven Plus, which showed an anomalous increase in the reported glucose of $180 \mathrm{mg} / \mathrm{dL}$ compared with the plasma glucose 90 min after the administration of the acetaminophen. The temporal profile of the erroneous CGM signal coincided with and followed the temporal profile of ISF and plasma acetaminophen profiles. Although the magnitude of the anomalous increase in the reported glucose was less for the G4 Platinum and the Medtronic REAL-time Guardian systems, both the G4 Platinum CGM and the Medtronic REAL-time Guardian CGM systems read higher than the baseline signal $(30 \mathrm{mg} / \mathrm{dL}$ and $21 \mathrm{mg} / \mathrm{dL}$, respectively). Because glucose detection with CGM probes relies on an electrochemical reaction, certain pharmaceuticals that are small molecules could interfere with CGM glucose detection especially if (a) their volume of distribution is large enough for the compound to reach the ISF and (b) the oxi- dation voltage is smaller than the working voltage used in CGM devices. Acetaminophen is a representative example of a commonly used medication that possesses such properties that increases its likelihood of interference with CGM glucose, which makes CGM interference testing even more of a priority for improving overall care of patients using CGM devices.

CGM manufacturers have two basic options for reducing the susceptibility of CGM sensors to electrochemical interferences. One method for reducing the susceptibility to pharmacologic interferences is to change the working voltage of the sensor. The FreeStyle ${ }^{\circledR}$ Navigator ${ }^{\circledR}$ developed by Abbott Diabetes Care (Alameda, CA) using the wired-enzyme approach operated at a very low applied voltage and was not susceptible to common pharmacologic interferences such as acetaminophen. ${ }^{14}$ Although the FreeStyle Navigator is no longer available, the Abbott Libre glucose sensor uses the same basic electrochemistry and is not expected to be susceptible to acetaminophen interference. The other method for reducing the susceptibility to pharmacologic interferences is to apply a permselective membrane to the sensor surface that inhibits the diffusion of potential pharmacologic interferences into the interior of the sensor. This is presumably the approach taken more recently by Dexcom and Medtronic with their latestgeneration CGM systems. As new CGM systems are developed 
by the leading diabetes technology companies and as sensor use becomes more widespread, the need for independent assessment of sensor susceptibility to common pharmacologic interferences will be greater. Hopefully, studies by independent researchers will show that future CGM systems have continued to improve their accuracy and reliability even in the presence of known potential pharmacologic interferences.

\section{Acknowledgments}

We are deeply indebted to the research participants. Our sincere thanks to Barbara Norby, RN, and Cheryl Shonkwiler, RN, for the conduct of the studies, as well as the staff of the Mayo Clinic Center for Translational Science Activities Clinical Trials Research Unit, Pamela Reich (research assistant), Michael Slama, and Brent McConahey (both research technologists). All persons mentioned above are at the Endocrine Research Unit, Mayo Clinic, Rochester, MN. The work was supported by funding from the JDRF to R.B., grants DK29953 and DK90541 to R.B. and grants DK085516 and DK DP3 094331 to A.B. from the National Institutes of Health, and grant UL1 TR000135 from the National Center for Advancing Translational Science, a component of the National Institutes of Health.

\section{Author Disclosure Statement}

No competing financial interests exist.

S.V. assisted in the conduct of the study and data handling. T.P. contributed to the idea of acetaminophen interference to investigate this issue and assisted in manuscript writing/ editing. R.D. assisted with acetaminophen analyses. A.B. and R.B. are responsible for study design, data analyses, and manuscript writing/reviewing/editing. R.B. is the guarantor of this work, had full access to all the data, and takes full responsibility for the integrity of data and the accuracy of data analysis.

\section{References}

1. Peyser T, Dassau E, Breton M, et al.: The artificial pancreas: current status and future prospects in the management of diabetes. Ann N Y Acad Sci 2014;1311:102-123.

2. Christiansen M, Bailey T, Watkins E, et al.: A new-generation continuous glucose monitoring system: improved accuracy and reliability compared with a previous-generation system. Diabetes Technol Ther 2013;15:881-888.

3. Bailey TS, Ahmann A, Brazg R, et al.: Accuracy and acceptability of the 6-day Enlite continuous subcutane- ous glucose sensor. Diabetes Technol Ther 2014;16:277283.

4. Bailey TS, Chang A, Christiansen M: Clinical accuracy of a continuous glucose monitoring system with an advanced algorithm. J Diabetes Sci Technol 2015;9:209-214.

5. Kovatchev BP, Patek SD, Ortiz EA, et al.: Assessing sensor accuracy for non-adjunct use of continuous glucose monitoring. Diabetes Technol Ther 2015;17:177-186.

6. Dungan K, Chapman J, Braithwaite SS, et al.: Glucose measurement: confounding issues in setting targets for inpatient management. Diabetes Care 2007;30:403-409.

7. Tang Z, Louie RF, Payes M, et al.: Oxygen effects on glucose measurements with a reference analyzer and three handheld meters. Diabetes Technol Ther 2000;2:349-362.

8. Tang Z, Du X, Louie RF, et al.: Effects of $\mathrm{pH}$ on glucose measurements with handheld glucose meters and a portable glucose analyzer for point-of-care testing. Arch Pathol Lab Med 2000;124:577-582.

9. Gaines AR, Pierce LR, Bernhardt PA: Fatal Iatrogenic Hypoglycemia: Falsely Elevated Blood Glucose Readings with a Point-of-Care Meter Due to a Maltose-Containing Intravenous Immune Globulin. 2008. www.fda.gov/Biologics BloodVaccines/SafetyAvailability/ucm 155099.htm (accessed December 7, 2015).

10. Maahs DM, DeSalvo D, Pyle L, et al. Effect of acetaminophen on CGM glucose in an outpatient setting. Diabetes Care 2015;38:e158-e159.

11. Basu A, Dube S, Slama M, et al.: Time lag of glucose from intravascular to interstitial compartment in humans. Diabetes 2013;62:4083-4087.

12. Schiavon M, Dalla Man C, Dube S, et al.: Time lag of glucose from intravascular to interstitial compartment in humans: a modeling analysis [abstract]. Diabetes 2014;63(Suppl 1):A214.

13. Basu A, Dube S, Veettil S, et al.: Time lag of glucose from intravascular to interstitial compartment in type 1 diabetes. J Diabetes Sci Technol 2015;9:63-68.

14. McGarraugh G: The chemistry of commercial continuous glucose monitors. Diabetes Technol Ther 2009;11(Suppl 1): S17-S24.

E-mail: basu.rita@mayo.edu 